\title{
Management Strategy for a Patient with Coexistence of Meningioma and Paraophthalmic Aneurysm
}

\author{
Burak Kinali ${ }^{1}$, Mehmet Senoglu², Evren Sandal' ${ }^{2}$ Gauravjot Sandhu ${ }^{3}$ and Ali Karadag ${ }^{4}$ \\ ${ }^{1}$ Department of Neurosurgery, Kahta State Hospital, Adlyaman, Turkey \\ ${ }^{2}$ Department of Neurosurgery, Tepecik Research and Training Hospital, University of Health Sciences, Izmir, Turkey \\ ${ }^{3}$ Department of Neurosurgery, Menemen State Hospital, Izmir, Turkey \\ ${ }^{4}$ Department of Neurology, University of South Dakota, Vermillion, SD, USA
}

\begin{abstract}
The coexistence of intracerebral aneurysm and meningioma is a rare occurrence. There is no consensus about the treatment options. We aim to present a case of a 61-year female admitted to this clinic with persistent headache and loss of vision in both eyes, especially on the left. Radiological images demonstrated a para-ophthalmic saccular aneurysm coexisting with an extraaxial homogenously enhancing mass in the right cavernous sinus, which was possibly a meningioma. A flow diverting stent, following the coil embolisation of the aneurysm, was placed. Then the patient underwent stereotactic radiosurgery for the cavernous sinus meningioma. Diagnosis and treatment of such pathologies are easily achieved with the technological advancements of imaging techniques. The hypothesis of increased hemodynamic pressures, due to meningioma causing intracranial aneurysms, may explain this coexistence. Endovascular surgery, followed by stereotactic radiosurgery, may be a reliable approach for a patient with coexistent meningioma and aneurysm.
\end{abstract}

Key Words: Meningioma, Aneurysm, Cavernous sinus.

How to cite this article: Kinali B, Senoglu M, Sandal E, Sandhu G, Karadag A. Management Strategy for a Patient with Coexistence of Meningioma and Paraophthalmic Aneurysm. J Coll Physicians Surg Pak 2021; 31(05):585-587.

\section{INTRODUCTION}

The coexistence of intracerebral aneurysm and tumor is a rare occurrence. It may be associated with pituitary adenomas, gliomas and metastatic tumors, mostly meningiomas. ${ }^{1}$ In the literature, many researchers have reported about the association of meningiomas and intracranial aneurysms. ${ }^{2}$ As there is still no consensus about the proper approach for this coexistence, there is a big challenge of diagnosis and treatment depending on the localisation. We report an uncommon case with a meningioma in the right cavernous sinus and left paraophthalmic saccular aneurysm, which was treated by endovascular approach followed by stereotactic radiosurgery.

\section{CASE REPORT}

A 61-year female was admitted to this clinic with persistent headache and dizziness. On physical examination, she had a significant loss of left eye vision and a partial right eye superior nasal field visual deficit.

Correspondence to: Dr. Mehmet Senoglu, Department of Neurosurgery, Tepecik Research and Training Hospital, University of Health Sciences, Izmir, Turkey

E-mail: mehmetsenoglu@hotmail.com

Received: September 08, 2019; Revised: December 25, 2019;

Accepted: January 02, 2020

DOI: https://doi.org/10.29271/jcpsp.2021.05.585
She did not have any other neuropathological symptoms. The head magnetic resonance imaging (MRI) showed a right cavernous extra-axial mass lesion. The mass size was $32 \times 23 \times 22 \mathrm{~mm}$. It had a homogeneous characteristic. Moreover, a $14 \times 12 \mathrm{~mm}$ hypointense lesion in the left prechiasmatic area, compressing the optic nerve, was detected. Visual impairment on the leftside was thought to be associated with this compression. The tumoraneurysm complex included a lesion of cavernous sinus and a paraophthalmic aneurysm (Figure 1 A-F). Computerised tomography angiography (CTA) showed a saccular aneurysm of theparaophthalmic segment of the internal carotid artery (ICA) (Figure $1 \mathrm{~A}-\mathrm{B}$ ). As the aneurysm was intact and had a smooth relationship with the medial wall of the tumor, it was planned to treat the patient by using the endovascular method to avoid the risk of aneurysm rupture. Coil embolisation with flow diverter stent was preferred (Figure 1 C-D-G-H). The patient was discharged after four days with good condition and no complications. Stereotactic radiosurgery (SRS) for the tumor of the cavernous sinus it was planned in the first month of follow-up of the endovascular procedure. Stereotactic reference was aimed by cone beam computed tomography (CBCT). Leksell gamma knife Icon was used for the procedure. G-shaped stereotactic frame was attached. The optic system was preserved by exposing to a maximum of $10 \mathrm{~Gy}$ and a minimum of $2.8 \mathrm{~Gy}$ with a median of $7.1 \pm 1.4$ Gy irradiation (Figure $2 \mathrm{~A}, \mathrm{~B}$ ). The patient did not have any post-procedural complications. After one year follow-up, no significant change was observed in the size and 
form of the aneurysm and tumor. Besides, visual impairment was partially recovered (Figure 2C,D).

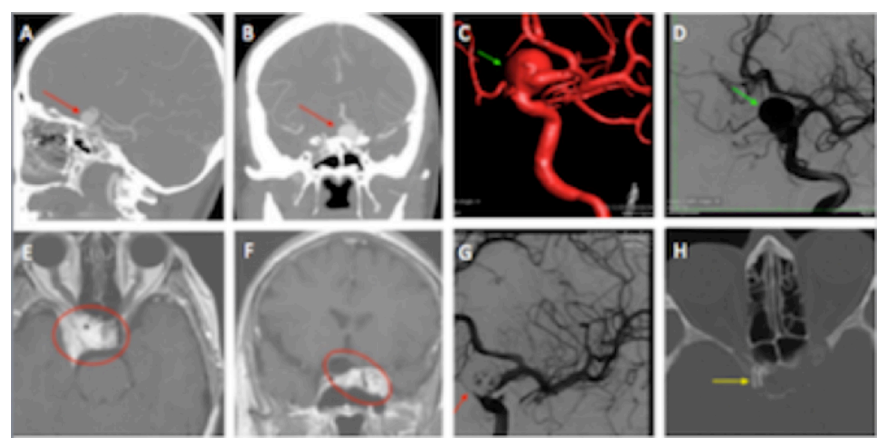

Figure 1: CT angiography shows a $15 \mathrm{~mm}$ saccular aneurysm in the paraophthalmic region (A, B) (red arrow). T1-weighted MRI images demonstrated an extra-axial homogenously enhanced mass in right cavernous sinus with close relation to aneurysm (E, F) (red circle). DSA showing para-ophtalmic sacculer aneurysm (C, D) (green arrow). Postoperative image of coil embolisation during DSA (G) (red arrow). Postoperative CT showing flow diverterstent in ICA (H) (yellow arrow).

(CT: Computerised tomography; MRI: Magnetic resonance imaging; DSA: Diagnostic substraction angiography).
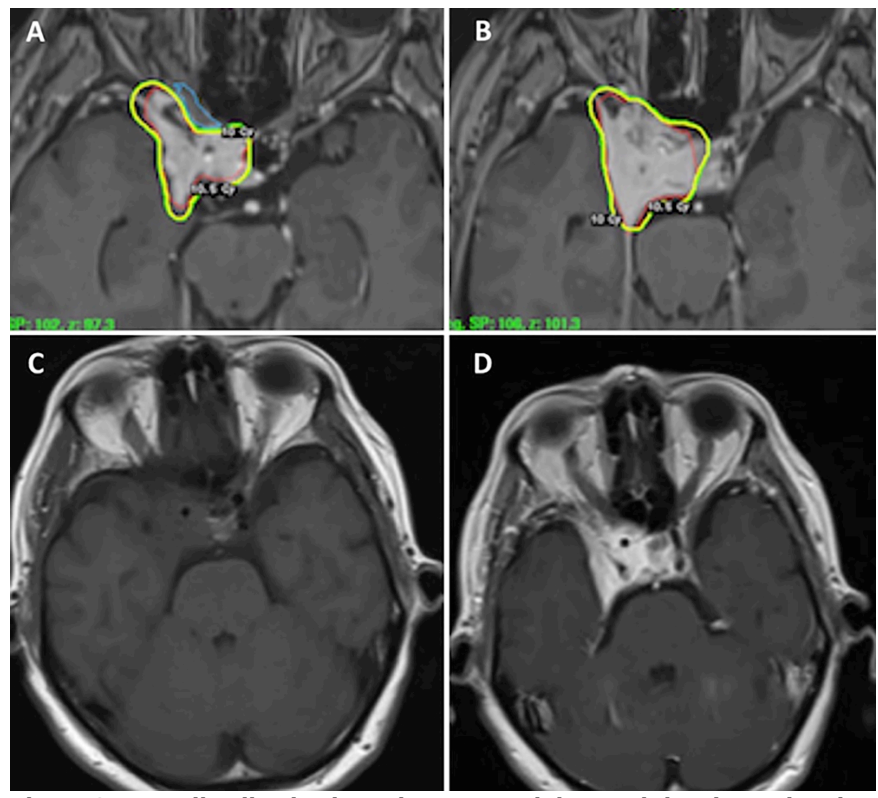

Figure 2: Dose distribution based on MRI . Axial T1-weighted MRI showing a typical meningioma in cavernous sinus with homogenous contrast enhancement and dural tail sign. Isodose lines are as follows: $10 \mathrm{~Gy}, 10.5$ Gy (A, B). Postoperative axial MRI sequences showing no significant change in meningiomasize (C,D).

(MRI: Magnetic resonance imaging, Gy, gray unit; CT: Computerised tomography; CTA: Computerised tomography angiography; CBCT: Cone beam computed tomography; DSA: Diagnostic substraction angiography; ICA: Internalcarotidartery; MRA:Magneticresonanceangiography;SRS:Stereotactic radiosurgery).

\section{DISCUSSION}

The true incidence of coexistence of the intracranial tumor and aneurysm may be underestimated as we do not perform magnetic resonance angiography (MRA), CTA or four-vessel-angiography routinely. Meningiomas are frequent intracerebral tumors comprising between $29.3 \%$ and $44 \%$ of all intracranial neoplasms. They may be associated with intracranial aneurysms. The coexistence of meningioma and aneurysm is reported between 0.3 and $1.1 \%{ }^{2-4}$

Many hypotheses regarding the mechanism of aneurysm's formation in meningioma are reported in the literature. Pia et al. argued that this relation might be due to increased local blood flow to the tumor; whereas, this might not explain the relation with contralateral aneurysms. ${ }^{4}$ Kandel et al, reported that it may be due to damage of arterial wall caused by meningioma, as they saw it during the surgical procedure for an aneurysm encased by a meningioma in the middle cerebral artery region. ${ }^{5}$ In this study, we report a case that presented with a left-sided cavernous meningioma in close association with a right-sided para-ophthalmic saccular aneurysm that may have occurred due to independent pathologies or intracranial hemodynamic changes. ${ }^{6}$

There are several treatment approaches for such pathologies in the literature, which are: endovascular, stereotactic and microsurgical methods ${ }^{1,2}$ According to Lee et al., 5 -year survival rate is between $86 \%$ and $99 \%$ and 10 -year survival is between $69 \%$ and $97 \%$ in cavernous sinus meningiomas. They suggested that it might be better to perform a resection when the tumor size exceeds $30 \mathrm{~mm}$. Javadopour etal. and O'Neil et al. reported that first endovascular coiling should be performed, and next, the microsurgical excision of the tumor. ${ }^{2,7}$ Hoy et al. reported a ruptured internal carotid artery aneurysm within a clinoidal meningioma, which occured two months after the SRS. ${ }^{8}$ Due to bilateral visual loss and the close association of the tumor with the aneurysm, we performed a double step approach with endovascular treatment followed by SRS. As there is a risk of rupture of the aneurysm after radiosurgery due to tumor size changes, we first performed the endovascular surgery.

The coexistence of intracranial tumor and aneurysm is important to know in the preoperative stage. The optimal treatment of such dual pathology is still controversial. The main goal should be to treat the pathological symptoms. In addition, the approach should be individualized, based on the location of aneurysm in relation to the tumor and surgical experience. We believe a stepwise approach with endovascular surgery followed by SRS is an efficient and safe method in selected cases.

\section{CONFLICT OF INTEREST:}

The authors declared no conflict of interest.

\section{AUTHORS' CONTRIBUTION:}

BK: Written the manuscript, literature search, collected the data.

MS: Conceived the idea, edited the manuscript.

ES: Literature search and collected the data.

GS, AK: Revised the manuscript.

\section{REFERENCES}

1. Fischer Br, Palkovic $S$, Holling M, Niederstadt $T$, Jeibmann $A$, Wassmann $\mathrm{H}$. Coexistence of cerebral aneurysm and meningioma - pure accident? Clin Neurol Neurosurg 2009; 
111(8):647-54. doi: 10.1016/j.clineuro.2009.05.016.

2. Javalkar V, Guthikonda B, Vannemreddy P, Nanda A. Association of meningioma and intracranial aneurysm: Report of five cases and review of literature. Neurol India 2009; 57(6):772-6. doi: 10.4103/0028-3886.59475.

3. Handa J, Matsuda I, Handa H. Association of brain tumor and intracranial aneurysms. Surg Neurol 1976; 6(1):25-9.

4. Pia HW, Obrador S, Martin JG. Association of brain tumours and arterial intracranial aneurysms. Acta Neurochir (Wien) 1972; 27(3):189-204. doi: 10.1007/BF01401881.

5. Kandel E, Ludkovskaya I, Dobjansky N. Aneurysm inside meningioma. Case report. Acta Neurochir (Wien)1986; 81(1-2):72-6. doi: 10.1007/BF01456268.

6. Lee Cc, Trifiletti Dm, Sahgal A, Desalles A, Fariselli L,
Hayashi M, et al. Stereotactic radiosurgery for benign (World Health Organization Grade I) cavernous sinus meningiomas-international stereotactic radiosurgery society (ISRS) practice guideline: A systematic review. Neurosurgery 2018; 83(6):1128-42. doi: 10.1093/neuros/ nyy009.

7. Javadpour M, Khan AD, Jenkinson MD, Foy PM, Nahser HC. Cerebral aneurysm associated with an intracranial tumour: Staged endovascular and surgical treatment in two cases. $\mathrm{Br} J$ Neurosurg 2004; 18(3):280-4. doi: 10.1080/02688 690410001732751.

8. Hoya K, Yoshimoto $Y$, Shin M, Nemoto S. Rupture of an internal carotid artery aneurysm within a clinoidal meningioma following stereotactic radiosurgery. Acta Neurochir (Wien) 2011; 153(10):1995-6. doi: 10.1007/ s00701-011-1095-1. 